\title{
Evaluation of clinical risk factors for developing pleural empyema secondary to liver abscess
}

\author{
Eunjue $\mathrm{Yi}^{1 \dagger}$, Tae Hyung $\mathrm{Kim}^{2 \dagger}$, Jun Hee Lee ${ }^{1}$, Jae Ho Chung ${ }^{1}$ and Sungho Lee ${ }^{1^{*}}$
}

\begin{abstract}
Background: The aim of this study was to investigate the clinical manifestation and predictive risk factors of pleural empyema developing during treatment of the pyogenic liver abscess.

Methods: Medical records of patients with the liver abscess in our institution were reviewed retrospectively. Enrolled patients were classified into four groups; Group 1: patients without pleural effusion, Group 2: patients with pleural effusion and who were treated noninvasively, Group 3: patient with pleural effusion and who were treated with thoracentesis, and Group 4: patients with pleural effusion that developed into empyema. Patient characteristics, clinical manifestation, and possible risk factors in development of empyema were analyzed.

Results: A total of 234 patients was enrolled in this study. The incidence rate of empyema was $4.27 \%$ (10 patients). The mean interval for developing pleural effusion was $5.6 \pm 6.35$ days. In multivariate analysis, risk factors for developing pleural effusion included the location of the liver abscess near the right diaphragm (segment 7 and 8 , $\mathrm{OR}=2.30, p=0.048)$, and larger diameter of the liver abscess ( $\mathrm{OR}=1.02, p=0.042)$. Among patients who developed pleural effusions, presences of mixed microorganisms from culture of liver aspirates $(O R=10.62, p=0.044)$, bilateral pleural effusion ( $\mathrm{OR}=46.72, p=0.012)$ and combined biliary tract inflammation $(\mathrm{OR}=21.05, p=0.040)$ were significantly associated with the need for invasive intervention including surgery on effusion.

Conclusion: The location of the liver abscess as well as pleural effusion, elevated inflammatory markers, and combined biliary tract inflammation may be important markers of developing pleural complication in patients with pyogenic liver abscess.
\end{abstract}

Keywords: Liver abscess, Pleural empyema, Risk factors

\section{Background}

The etiology of pleural effusion (PE) varies from benign inflammatory disease to malignancy [1]; however, empyema is largely a result of preceding pneumonia, thoracic surgery, or chest injury [2]. More than half of bacterial pneumonia cases are associated with parapneumonic PE [3]; the presence of alcoholism, leukocytosis $\left(>15,000 \mathrm{~mm}^{3}\right)$, or neutrophilia, ( $>50 \%$ of counted leukocytes); or being male, all of which have been reported as predictive risk factors for empyema $[4,5]$. Although it is uncommon,

\footnotetext{
* Correspondence: sholeemd@korea.ac.kr

${ }^{\dagger}$ Eunjue Yi and Tae Hyung Kim contributed equally to this work.

${ }^{1}$ Department of Thoracic and Cardiovascular Surgery, Korea University

College of Medicine, 73, Goryeodae-ro, Seongbuk-gu, Seoul 02841, Republic

of Korea

Full list of author information is available at the end of the article
}

pyogenic liver abscess (PLA) substantially increases the risk of empyema by 18 times [6].

Pleural empyema following PLA is a rare but challenging condition that negatively impacts on the treatment process. Both diseases may require surgical intervention, with high risk of mortality and morbidity [2, 7]. In the past, pleural empyema combined with amoebic liver abscess has been reported sporadically. With the etiologic shift, Klebsiella pneumoniae is likely to occasionally be a causative agent [8].

Several studies have investigated the risk of metastatic infections following PLA, and showed that diabetes, alcoholism, and bacteremia could be independent risk factors for the metastatic infections [9]. K. pneumonia was associated with a higher incidence of extra-hepatic

(C) The Author(s). 2019 Open Access This article is distributed under the terms of the Creative Commons Attribution 4.0 International License (http://creativecommons.org/licenses/by/4.0/), which permits unrestricted use, distribution, and 
infections. However, there are few reports evaluating the risk of pleural empyema in association with PLA. Goumard et al. reported that his team had conducted the first study of pleural empyema followed by liver resection surgery [10]. In this study, right sided hepatic resection, intraabdominal sepsis along with postoperative bile leakage or history of diaphragm opening could be risk factors.

During the past decade, we have treated several empyema cases associated with PLA. Based on these cumulated experiences, we investigated and analyzed the possible risk factors of pleural empyema in patients with PLA, such as the presence and location of pleural effusion or microbiology. Early detection of empyema is important because optimal surgical intervention is essential for better treatment outcomes [11]. Predictive cautions could facilitate the therapeutic process of PLA with few sequelae.

\section{Methods}

\section{Patient characteristics and initial clinical conditions}

Medical records of patients who had been admitted and treated for PLA between October 2008 and December 2017 in our institution were reviewed retrospectively. This study has been approved by Institutional Review Board of Korea University Anam Hospital (IRB Number; 2019AN0183) and performed in accordance with the ethical guidelines of the 2008 Declaration of Helsinki. A waiver of informed consent was obtained.

Inclusion criteria were (1) patients older than 18, (2) patients diagnosed with PLA, and (3) patients who underwent percutaneous drainage and antibiotic administration. A total of 290 patients was candidates for this study; patients who had hepatocellular carcinoma or had received transarterial chemoembolization (TACE) for hepatocellular carcinoma (40 patients) and patients with cholangiocarcinoma (16 patients) were excluded.

Demographic characteristics were investigated through patient interview records. Smoking status was categorized into never (never smoked or smoked 100 or fewer cigarettes ever), former (smoked at least 100 cigarettes but had quit at the time of the interview), and current smoker (smoked at least 100 cigarettes and currently smoking) [12]. Amount of alcohol consumption was classified into none, mild (less than two drinks per week), moderate (more than two and less than five drinks per week), and excessive (five or more drinks per week). One drink was defined as consumption of $200 \mathrm{ml}$ of a beverage with $15 \%$ alcohol content [13].

Presence of major comorbidities was described, and comorbidity scores were calculated according to the modified Charlson Comorbidity Index [14, 15]. Combined metastatic infectious conditions were identified according to the radiologic reports of chest and abdominal CT studies. The amount of ascites was estimated using abdominal $\mathrm{CT}$ and ultrasonographic examinations. The presence of combined biliary tract inflammation was defined as the presence of cholangitis, cholecystitis, choledocholithiasis, or other inflammatory condition observed in imaging studies at the time of PLA diagnosis.

The researchers also investigated the admission route of the patients, whether emergency (ED) or outpatient department. They also checked the units to which patients were first admitted-general ward or intensive care unit (ICU). The initial admission route and place could be important for indirectly estimating clinical severity. Demographic information and initial clinical data are described in Table 1.

\section{Study group categorization and clinical manifestation}

Detection of PE was dependent on the radiologic findings. The location of PE was described as the site at which PE first appeared-right, left, or bilateral. Intervals of PE were defined as the periods between diagnosis of PLA and detection of PE. The authors defined complicated PE as biochemical analyses that satisfied at least one of the following conditions: (1) $\mathrm{pH}<7.20$, (2) lactate dehydrogenase $>1000 \mathrm{IU} / \mathrm{L}$, and (3) glucose $<60 \mathrm{mg} / \mathrm{dL}$ [3]. Pleural empyema was defined by performance of closed thoracostomy or video-assisted thoracoscopic surgery (VATS) drainage. When the PE disappeared spontaneously without any invasive procedures (thoracentesis for diagnostic and treatment purposes, chest tube insertion, and VATS drainage) during the treatment periods, we labeled this simple pleural effusion.

Patients included in this study were classified into PE (-), Group 1 and PE (+) groups. PE (+) groups were subdivided by treatment course into three groups. (1) Group 2: patients with simple and uncomplicated PE, (2) Group 3: patients with complicated PE who underwent thoracentesis, and (3) Group 4: patients with empyema. Clinical features of PLA, $\mathrm{PE}$, laboratory findings, and treatment results of each group were investigated.

The location and numbers of PLA were identified using abdominal CT and ultrasonography. The locations were described in four ways, (1) segmental: one to eight segments, (2) sectional: left lateral, left medial, right anterior, and right posterior, (3) according to the relationship with the diaphragm (near right, near left, and nonrelated), and (4) according to lobe (right or left). The longest diameter of the PLA was measured. In cases of multiple PLA, the location and diameter of the largest were described. The microbiologic data of the PLA were based on culture reports performed on the pus obtained from percutaneous drainage. Microorganisms of the PLA were categorized into Klebsiella pneumoniae only, other single aerobic microorganisms such as Escherichia coli or streptococcus, mixed, and others including anaerobes. 


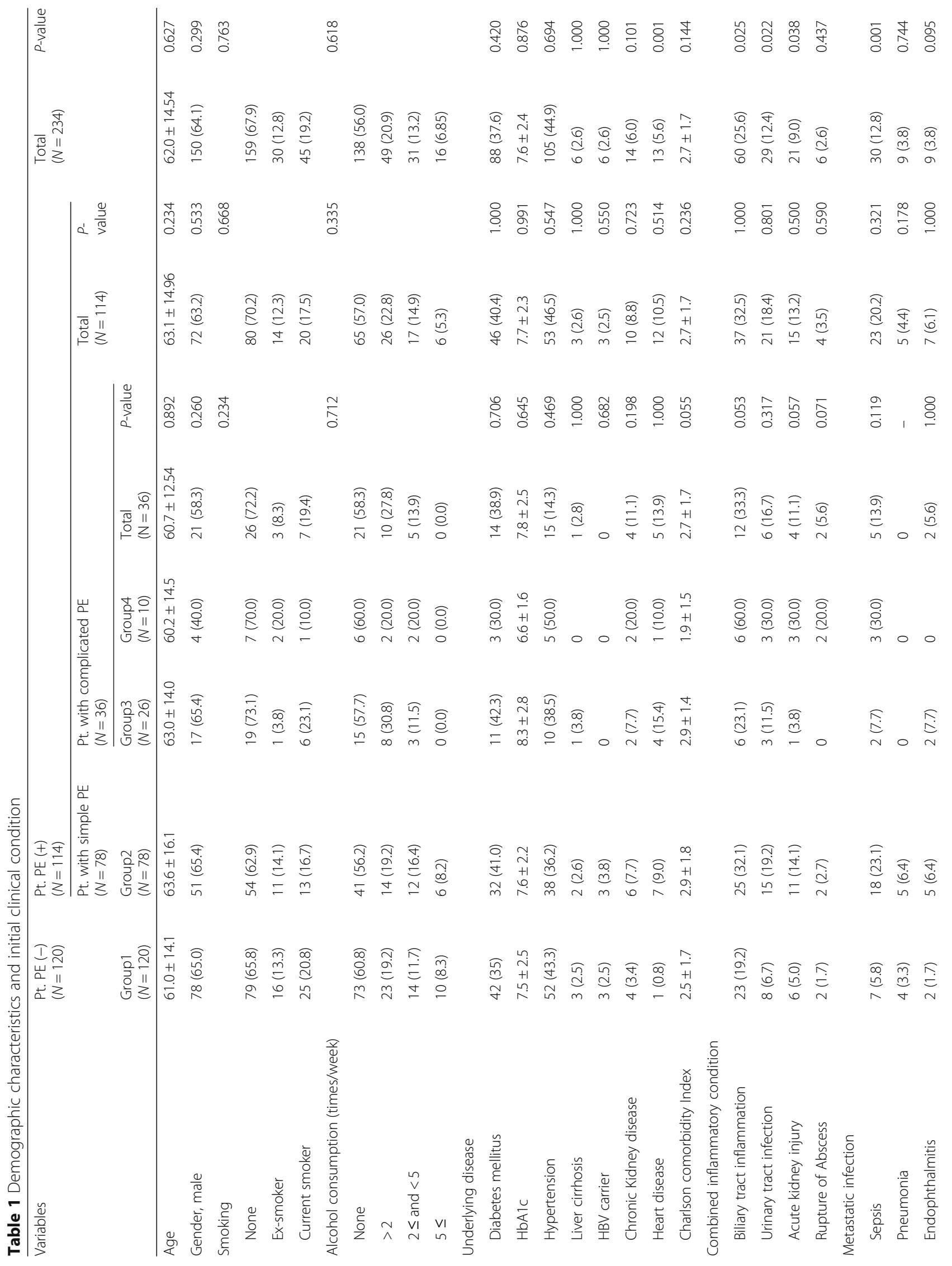




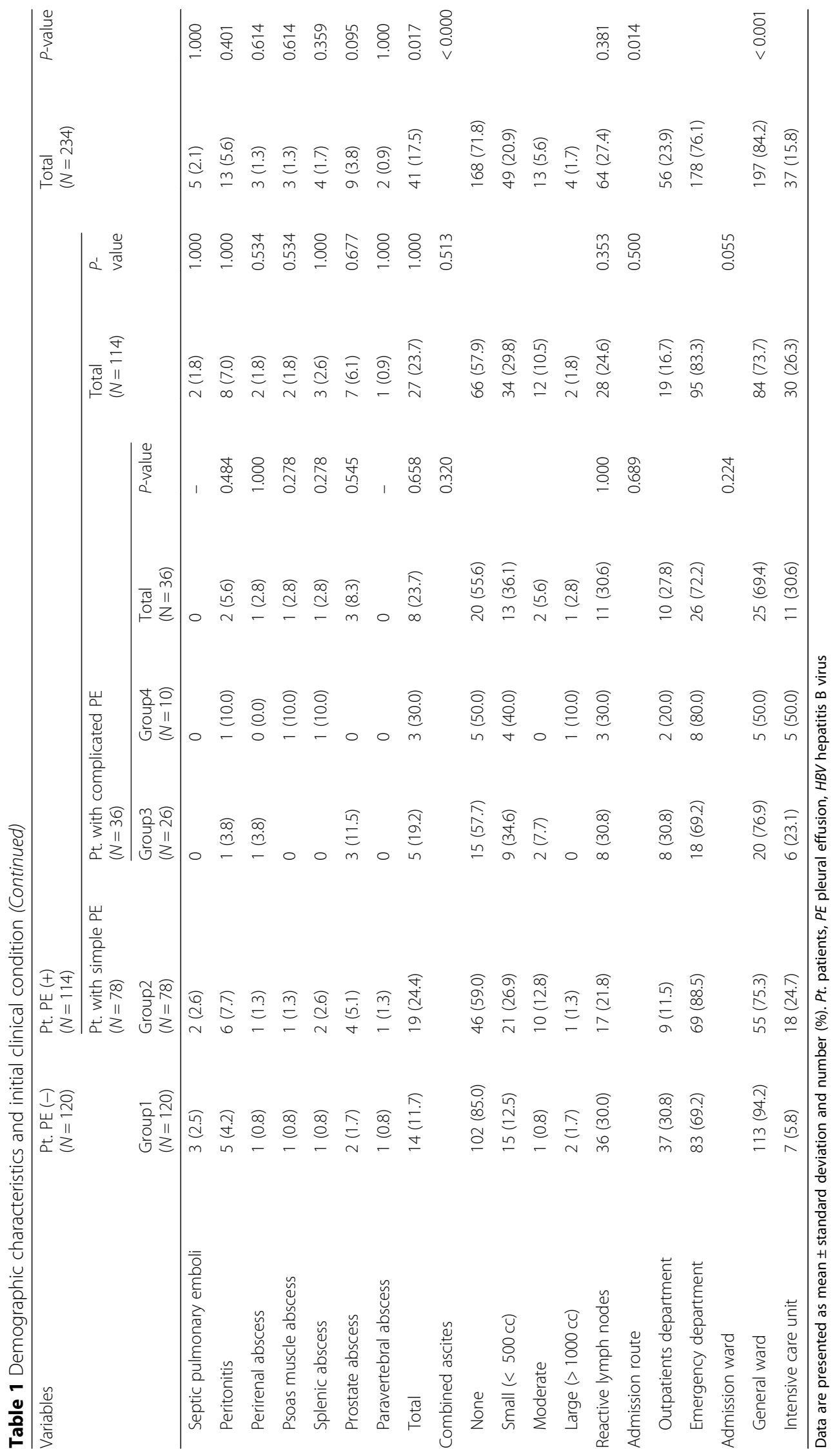




\section{Statistical analysis}

Clinical data were analyzed using $\mathrm{IBM}^{\circ} \mathrm{SPSS}^{\circ}$ Statistics software Version 22.0 (IBM, Armonk, NY, USA). A chisquare test and Fisher's exact test were used for analysis of categorical data. Student's $t$-test and Mann-Whitney tests were used for continuous data.

Univariate analysis using a logistic regression model for identifying risk factors of PE between PE negative (Group 1) and PE positive (Groups 2, 3, and 4) patients was performed. Identical analysis was performed between Group 2 and patients with PE requiring intervention (thoracentesis, chest tube insertion, or surgery, Groups 3 and 4). Then, analysis was performed between Groups 3 and 4. Multivariate analysis was performed based on the results of univariate analysis. Statistical significance was defined as a $p$ value less than 0.05 .

\section{Results}

\section{Demographic characteristics and initial clinical} information

The study population was comprised of 234 patients. A total of $120(51.3 \%)$ patients did not present with PE during the treatment period. Among the patients with PE (114 patients, 48.7\%), 36 needed invasive intervention, and 10 (8.8\% of PE positive patients) suffered from pleural empyema. Clinical manifestation of abscess, laboratory findings, and treatment results of each group are presented in Tables 2 and 3.

\section{Risk factors of pleural effusion and need for interventional methods including surgery}

The mean interval of developing pleural effusion was $5.6 \pm 6.35$ days. Multivariate analysis demonstrated that elevated CRP level at the second week post-PLA diagnosis $(p=0.001)$, location near the right diaphragm (segments 7 and $8, p=0.048$ ), and larger liver abscess diameter $(p=0.042)$ were statistically significant risk factors of pleural effusion (Table 4).

Risk factors for interventions including thoracentesis, chest tube insertion, and surgery comprised microbial culture results from pus drained from the PLA. When the isolated organisms were mixed with several types of gram positive and negative species, the hazard ratio of need for intervention increased to $10.62(p=0.044)$.

Pleural empyema developed in 10 patients $(4.3 \%$ in total patients, $8.8 \%$ in PE positive patients, and $27.8 \%$ in invasively treated patients). Five patients were cured by chest tube insertion, and five patients required surgery (three patients underwent initial chest tube drainage, and two patients underwent initial VATS drainage without chest tube insertion). Risk factors of empyema in patients who needed interventions were presence of combined biliary tract inflammation $(p=0.004)$ and bilateral pleural effusion $(p=0.012)$. The results of univariate and multivariate analysis are summarized in Additional file 1: Table S1 and Table 4.

\section{Discussion}

Out of 234 PLA patients in the past decade, we noted 10 cases of pleural empyema, with five cases needing surgical decortication. The estimated incidence rate was very low, only $4.3 \%$, and it seemed difficult to evaluate predictive risk factors directly through multivariate analysis. Therefore, we started by investigating risk factors of PE to identify the possible conditions that contribute to pleural empyema.

When the enrolled patients were divided into PE $(-)$ and PE $(+)$ groups, multivariate analysis revealed three statistically significant risk factors: (1) location of PLA near the right diaphragm (segments 7 and 8), (2) larger abscess size, and (3) elevated CRP level at the second week post- diagnosis. However, considering that the median interval from diagnosis of PLA to appearance of PE was 5.6 days ( \pm 6.35 , ranging 4.4 to 6.7), the increased numbers might be explained as results rather than causes. As the estimated hazard ratio of larger abscess size was 1.02, this may have little effect on PE.

Patients with PLA located near the right diaphragm were at higher risk-more than double-of developing pleural effusion. Hydrothorax-associated hepatic diseases have been reported as largely dependent of right sides, because the ascites moves through diaphragmatic defects along pressure gradients [16]. The occurrence of pleural empyema after liver resection is also known to be primarily related to right hepatectomy [11]. There were reports that upper abdominal surgery induced significant postoperative changes in the surface electromyogram in the diaphragm [17]. Thus, irritation due to surgery or inflammation near the right diaphragm is a possible cause of PE.

Although PE had appeared, it will not harmful if it does not developed into empyema. In our study, 31.6\% of simple PE progressed to complicated cases (36 of 114 patients), and multivariate analysis revealed that presence of mixed gram (+) and gram (-) microorganisms in the culture of pus drained from the liver abscess was a significant risk factor $(\mathrm{HR}=10.62)$. K. pneumoniae, which is the most common etiology of PLA [6] and is frequently complicated by metastatic infections such as bacteremia, sepsis, endophthalmitis, pulmonary infection, or intraabdominal abscess [8], was not associated with increased risk for complicated PE $(p=0.697)$. Complicated PE is often related to pneumonia [3], which can manifest as a metastatic infection in $12.6 \%$ of PLA patients [6]. However, presence of pneumonia was not significantly associated with development of complicated PE in our study $(p=0.178)$. 


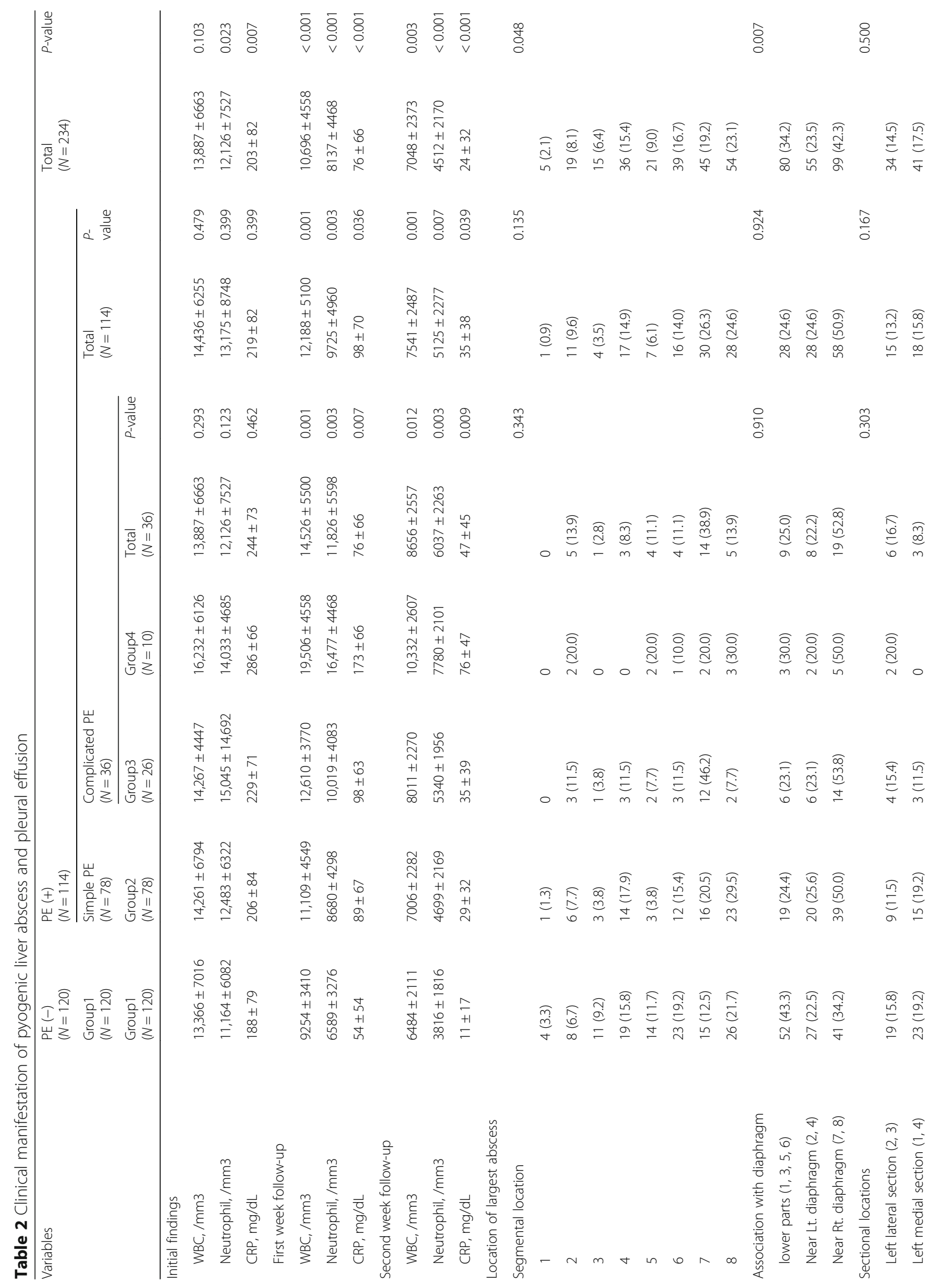




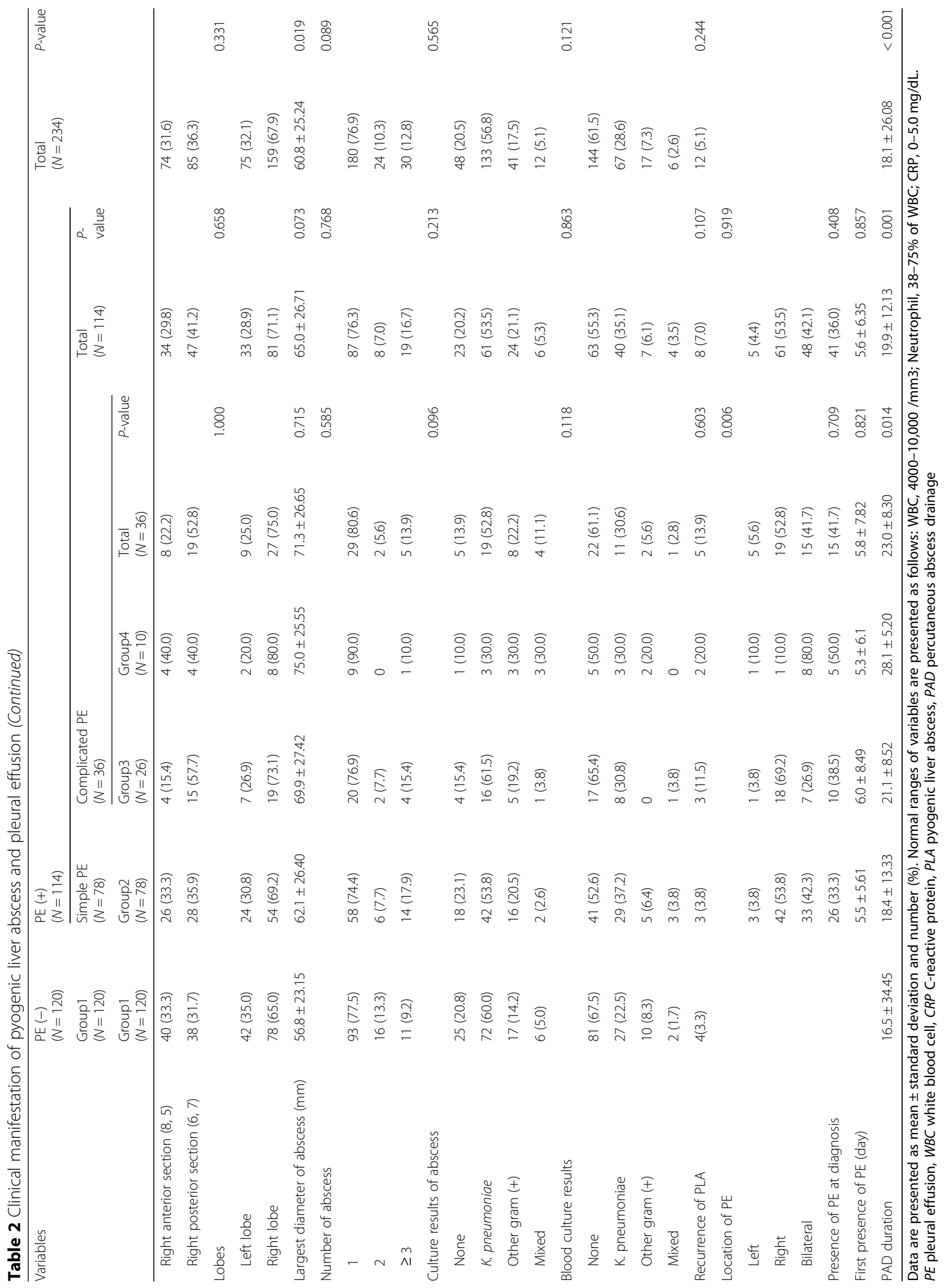




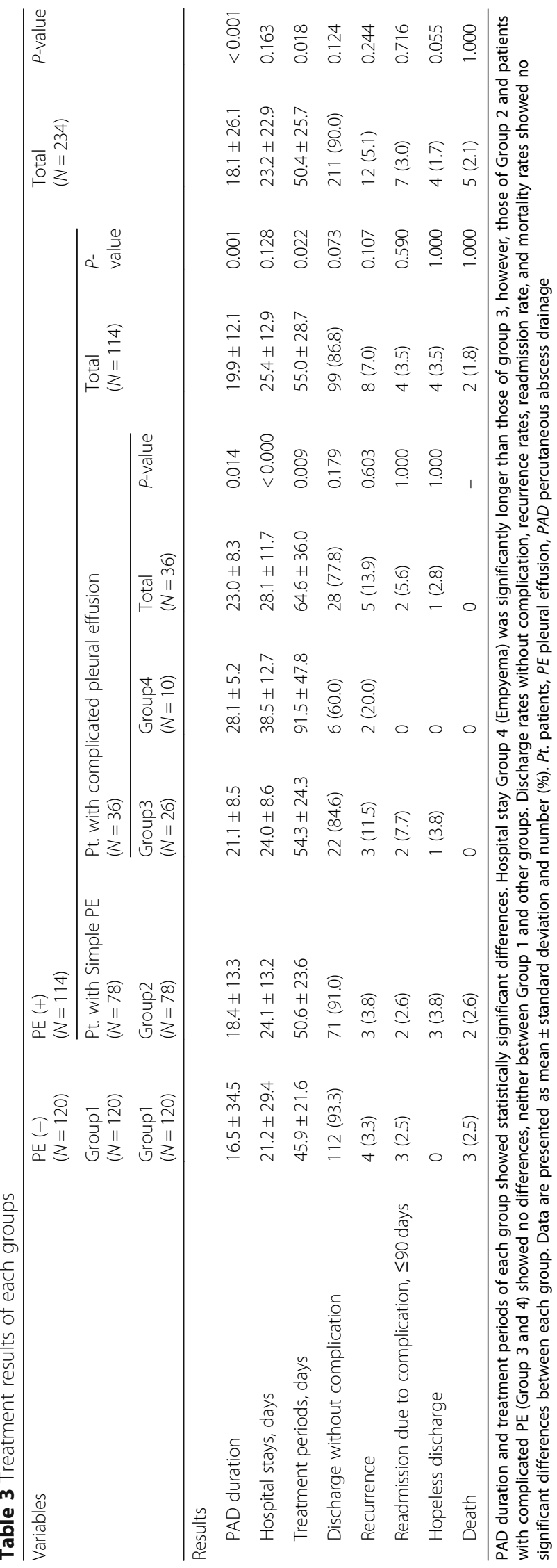


Table 4 Multivariate analysis for investigating risk factors of developing PE, complicated PE and pleural empyema

\begin{tabular}{|c|c|c|c|}
\hline Variables & OR & $\begin{array}{l}95 \% \text { Confidential } \\
\text { Interval }\end{array}$ & $P$-value \\
\hline \multicolumn{4}{|c|}{ Multivariate analysis for risk factors of PE } \\
\hline Heart Disease & 2.88 & $0.000-0.000$ & 0.998 \\
\hline Admission via ER & 1.69 & $0.688-4.149$ & 0.252 \\
\hline Admission to ICU & 1.98 & $0.568-6.900$ & 0.284 \\
\hline Biliary tract inflammation & 1.78 & $0.757-4.198$ & 0.186 \\
\hline Urinary tract infection & 1.70 & $0.560-5.150$ & 0.349 \\
\hline Acute kidney injury & 1.85 & $0.434-7.863$ & 0.406 \\
\hline Sepsis & 2.60 & $0.688-9.851$ & 0.159 \\
\hline Total metastatic infections & 0.64 & $0.213-1.894$ & 0.416 \\
\hline Combined ascites & & & 0.352 \\
\hline small & 1.42 & $0.573-3.541$ & 0.447 \\
\hline moderate & 6.80 & $0.705-65.598$ & 0.097 \\
\hline large & 0.79 & $0.060-10.519$ & 0.860 \\
\hline $\begin{array}{l}\text { Second week follow-up CRP, } \\
\mathrm{mg} / \mathrm{dL}\end{array}$ & 1.04 & $1.016-1.061$ & 0.001 \\
\hline $\begin{array}{l}\text { Location associated with } \\
\text { diaphragm }\end{array}$ & & & 0.137 \\
\hline Near left diaphragm $(2,4)$ & 1.72 & $0.694-4.259$ & 0.242 \\
\hline Near right diaphragm $(7,8)$ & 2.30 & $1.008-5.265$ & 0.048 \\
\hline Largest diameter of abscess (mm) & 1.02 & $1.001-1.034$ & 0.042 \\
\hline \multicolumn{4}{|c|}{ Multivariate analysis for risk factors of complicated PE } \\
\hline Admission via ER & 0.41 & $0.120-1.378$ & 0.148 \\
\hline Initial CRP, mg/dL & 1.01 & $0.999-1.013$ & 0.090 \\
\hline 1st week follow-up CRP, mg/dL & 1.00 & $0.990-1.008$ & 0.830 \\
\hline 2nd week follow-up CRP, mg/dL & 1.01 & $0.993-1.030$ & 0.236 \\
\hline \multicolumn{4}{|l|}{ Culture results of abscess } \\
\hline K. pneumoniae & 1.35 & $0.296-6.170$ & 0.697 \\
\hline Other gram $(+)$ & 2.70 & $0.490-14.823$ & 0.254 \\
\hline Mixed & 10.62 & $1.069-105.411$ & 0.044 \\
\hline \multicolumn{4}{|c|}{ Multivariate analysis for risk factors of pleural empyema } \\
\hline Biliary tract inflammation & 21.05 & $1.152-384.785$ & 0.040 \\
\hline Urinary tract infection & 3.07 & $0.202-46.678$ & 0.419 \\
\hline \multicolumn{4}{|l|}{ Location of PE } \\
\hline Right & 12.63 & $0.249-640.051$ & 0.205 \\
\hline Bilateral & 46.72 & $2.354-927.452$ & 0.012 \\
\hline Association with diaphragm & & & 0.642 \\
\hline Near left diaphragm $(2,4)$ & 2.79 & $0.115-67.482$ & 0.528 \\
\hline Near right diaphragm $(7,8)$ & 0.50 & $0.047-5.348$ & 0.567 \\
\hline
\end{tabular}

$P E$ pleural effusion, $O R$ odds ratio, $E R$ emergency room, $I C U$ intensive care unit, CRP C-reactive protein. Normal ranges of variables are presented as follows: WBC, $4000-10,000 / \mathrm{mm} 3$; Neutrophil, $38-75 \%$ of WBC; CRP, $0-5.0 \mathrm{mg} / \mathrm{dL}$

This is likely to suggest that changes from simple to complicated PE are due to failure of antibiotic treatment, because the initial treatment choice of PLA is percutaneous drainage and third-generation cephalosporin with or without metronidazole [7]. Empirical antibiotic application could have effects on mainstream microorganisms but not for unusual and mixed species (Regimens and change rates of antibiotics in our study were described in Additional file 2: Table S2). No microorganisms were cultured from drained pleural effusion, including empyema; therefore, development of complicated PE might not be due to metastatic infection.

The etiology of PLA did not seem to influence development of empyema from complicated PE. Multivariate analysis revealed that combined biliary tract inflammation (choledocholithiasis, cholangitis, or cholecystitis) and presence of bilateral PE was significantly associated with pleural empyema (Univariate and multivariate analysis of risk factors for bilateral PE were described in Additional file 2: Table S3). Combined biliary tract inflammation has been reported as a common causative condition for PLA [7, 18]. A total of 60 patients $(25.6 \%)$ had combined biliary tract inflammation (cholangitis in 9, cholecystitis in 48, choledocholithiasis in six, one GB perforation and one cholangiohepatitis), 36 in $\mathrm{PE}(+)$ patients (25.2\%), 12 in complicated PE (33.3\%), and six in empyema cases $(60 \%)$ in the current study (Additional file 2: Table S4). The proportion of combined biliary tract inflammation was increased in empyema patients: this might indicate that obstruction of the biliary tract could deteriorate the liver abscess and then interfere with antibiotics and increased risks for empyema. The presence of bilateral PE might reflect relatively extensive effusion, which is seldom resolved spontaneously or treated by thoracentesis, and therefore could be aggravated.

This study had several limitations. First, because the study population was small, we had to evaluate risk factors indirectly. Second, we did not fully investigate antibiotic therapies due to the complexity of various combinations during the treatment period. For more accurate assessment of antibiotic effects, further examination is needed regarding antibiotic combinations and changes. Finally, more detailed analysis for initial inflammatory conditions in PLA patients, including laboratory tests, should be implemented. Because there were few patients who underwent regular laboratory test follow-up for identifying inflammatory conditions, including procalcitonin, we could not perform more precise analysis on laboratory findings. Initial and follow-up changes in inflammatory markers are important factors to tracing successful application of treatment strategies.

\section{Conclusions}

The location of liver abscess near the right diaphragm, detection of mixed microorganisms in pus drained from liver abscess, and combined biliary tract disease could 
affect the development of pleural complications requiring invasive procedures such as chest tube insertion and surgical debridement. In multivariate analysis, combined biliary tract inflammation and bilateral pleural effusion were identified as significant risk factors for pleural empyema.

\section{Supplementary information}

Supplementary information accompanies this paper at https://doi.org/10 1186/s12876-019-1128-4.

Additional file 1: Table S1. Univariate analysis for investigating risk factors of developing pleural effusion, complicated pleural effusion and empyema.

Additional file 2: Table S2. Antibiotics treatment regimen.

Table S3. Univariate and Multivariate analysis of risk factors for bilateral pleural effusion. Table S4. Classification of combined biliary tract disease according to the Groups.

\section{Abbreviations}

ICU: Intensive care unit; OR: Odds ratio; PE: Pleural effusion; PLA: Pyogenic liver abscess; TACE: Transarterial chemoembolization; VATs: Video-assisted thoracoscopic surgery

\section{Acknowledgements}

We would like to thank Editage (www.editage.co.kr) for English language editing.

\section{Author's contributions}

YE and KTH analyzed the collected data and wrote the manuscript. LSH conceptualized this study and reviewed the manuscript. LJH and CJH collected the data. All authors read and approved the final manuscript.

\section{Funding}

None.

\section{Availability of data and materials}

The datasets generated and/or analyzed during the current study are not publicly available due personal medical records, but are available from the corresponding author on reasonable request and approval of IRB.

\section{Ethics approval and consent to participate}

This study protocol and a waiver of informed consent has been approved by Institutional Review Board of Korea University Anam Hospital. (IRB Number; 2019AN0183).

\section{Consent for publication}

Not applicable.

\section{Competing interests}

I and my coauthors declare that we have no competing interests.

\section{Author details}

${ }^{1}$ Department of Thoracic and Cardiovascular Surgery, Korea University College of Medicine, 73, Goryeodae-ro, Seongbuk-gu, Seoul 02841, Republic of Korea. ${ }^{2}$ Department of Internal Medicine, Korea University College of Medicine, 73, Goryeodae-ro, Seongbuk-gu, Seoul 02841, Republic of Korea.

Received: 24 June 2019 Accepted: 22 November 2019

Published online: 16 December 2019

\section{References}

1. Porcel JM, Esquerda A, Vives M, Bielsa S. Etiology of pleural effusions: analysis of more than 3,000 consecutive thoracenteses. Arch Bronconeumol. 2014;50:161-5

2. Semenkovich TR, Olsen MA, Puri V, Meyers BF, Kozower BD. Current state of empyema management. Ann Thorac Surg. 2018;105:1589-96.
3. Ahmed AEH, Yacoub TE. Empyema thoracis. Clin Med Insights Circ Respir Pulm Med. 2010;4:1-8.

4. Zablockis R, Petruskeviciene R, Nargela RV. Causes and risk factors of pleural empyema and complicated parapneumonic pleural effusion. Medicina. 2010;46:113-9.

5. Falguera M, Carratalà J, Bielsa S, García-Vidal C, Ruiz-González A, Chica I, et al. Predictive factors, microbiology and outcome of patients with parapneumonic effusion. Eur Respir J. 2011;38:1173-9.

6. Keller JJ, Tsai MC, Lin CC, Lin YC, Lin HC. Risk of infections subsequent to pyogenic liver abscess: a nationwide population-based study. Clin Microbiol Infect. 2013:19:717-22

7. Heneghan HM, Healy NA, Martin ST, Ryan RS, Nolan N, Traynor O, et al. Modern management of pyogenic hepatic abscess: a case series and review of the literature. BMC Res Notes. 2011:4:80.

8. Lee SS, Chen YS, Tsai HC, Wann SR, Lin HH, Huang CK, et al. Predictors of septic metastatic infection and mortality among patients with Klebsiella pneumoniae liver abscess. Clin Infect Dis. 2008;47:642-50.

9. Chen SC, Lee YT, Lai KC, Cheng KS, Jeng LB, Wu WY, et al. Risk factors for developing metastatic infection from pyogenic liver abscesses. Swiss Med Wkly. 2006;136:119-26.

10. Goumard C, Fuks D, Cauchy F, Belghiti J, Paugam-Burtz C, Castier Y, et al. Pleural empyema following liver resection: a rare but serious complication. World J Surg. 2016;40:2999-3008

11. Chung JH, Lee SH, Kim KT, Jung JS, Son HS, Sun K. Optimal timing of thoracoscopic drainage and decortication for empyema. Ann Thorac Surg. 2014:97:224-9.

12. National Center for Health Statistics. https://www.cdc.gov/nchs/nhis/ tobacco/ tobacco_glossary.htm. Accessed 13 Dec 2018.

13. National Institute on Alcohol Abuse and Alcoholism. https://www.niaaa.nih. gov/alcohol-health/overview-alcohol-consumption/moderate-bingedrinking. Accessed 13 Dec 2018.

14. Charlson ME, Pompei P, Ales KL, MacKenzie CR. A new method of classifying prognostic comorbidity in longitudinal studies: development and validation. J Chronic Dis. 1987;40:373-83.

15. Deyo RA, Cherkin DC, Ciol MA. Adapting a clinical comorbidity index for use with ICD-9-CM administrative databases. J Clin Epidemiol. 1992;45:613-9.

16. Alonso JC. Pleural effusion in liver disease. Semin Respir Crit Care Med. 2010;31:698-705.

17. Berdah SV, Picaud R, Jammes Y. Surface diaphragmatic electromyogram changes after laparotomy. Clin Physiol Funct Imaging. 2002;22:157-60.

18. Ku CL, von Bernuth H, Picard C, Zhang SY, Chang HH, Yang K, et al. Selective predisposition to bacterial infections in IRAK-4-deficient children: IRAK-4-dependent TLRs are otherwise redundant in protective immunity. J Exp Med. 2007:204:2407-22.

\section{Publisher's Note}

Springer Nature remains neutral with regard to jurisdictional claims in published maps and institutional affiliations.

\section{Ready to submit your research? Choose BMC and benefit from:}

- fast, convenient online submission

- thorough peer review by experienced researchers in your field

- rapid publication on acceptance

- support for research data, including large and complex data types

- gold Open Access which fosters wider collaboration and increased citations

- maximum visibility for your research: over $100 \mathrm{M}$ website views per year

At $\mathrm{BMC}$, research is always in progress.

Learn more biomedcentral.com/submission 